\title{
Mixed cell therapy of bone marrow-derived mesenchymal stem cells and articular cartilage chondrocytes ameliorates osteoarthritis development
}

\author{
Indira Prasadam ${ }^{1,6}$, Akoy Akuien $^{1,6}$, Thor E Friis ${ }^{1}$, Wei Fang ${ }^{1,2}$, Xinzhan Mao ${ }^{1,3,4}$, Ross W Crawford ${ }^{1,5}$ and Yin Xiao
}

Of the many cell-based treatments that have been tested in an effort to regenerate osteoarthritic articular cartilage, none have ever produced cartilage that compare with native hyaline cartilage. Studies show that different cell types lead to inconsistent results and for cartilage regeneration to be considered successful, there must be an absence of fibrotic tissue. Here we report of a series of experiments in which bone marrow-derived stem cells (BMSCs) and articular cartilage chondrocytes (ACCS) were mixed in a 1:1 ratio and tested for their ability to enhance cartilage regeneration in three different conditions: (1) in an in vitro differentiation model; (2) in an ex vivo cartilage defect model implanted subcutaneously in mice; and (3) as an intra-articular injection in a meniscectomy-induced OA model in rats. The mixed cells were compared with monocultures of BMSCs and ACCs. In all three experimental models there was significantly enhanced cartilage regeneration and decreased fibrosis in the mixed BMSCs+ACCs group compared with the monocultures. Molecular analysis showed a reduction in vascularization and hypertrophy, coupled with higher chondrogenic gene expression resulting from the BMSCs+ACCs treatment. Together, our data suggest that mixed BMSCs + ACCs treatment is highly chondro-protective and is more effective in regenerating damaged cartilage in both the ex vivo cartilage defect and post-trauma OA disease models. The results from this approach could potentially be used for regeneration of cartilage in $\mathrm{OA}$ patients.

Laboratory Investigation (2018) 98, 106-116; doi:10.1038/labinvest.2017.117; published online 16 October 2017

Osteoarthritis (OA) is a chronic degenerative disease that affects articular cartilage surfaces of the joints. ${ }^{1}$ It is associated with aging and injuries and tends to affect the joints that have been continually stressed throughout life, including knees, hips, fingers, and lower spine. ${ }^{1}$ There have been various cellbased methods available clinically to correct cartilage degeneration in OA patients. These strategies include: (i) cartilage replacement techniques which compromised of chondrocyte autograft transfer (OAT) and osteochondral graft transplantation and (ii) cartilage regeneration techniques such as autologous chondrocytes injection (ACI), matrixinduced autologous chondrocyte implantation (MACI) and bone marrow stimulation by a microfracture technique. ${ }^{2}$ However, the success rate for these treatments have been inconsistent, involved invasive procedure causing donor site morbidity and their long-term benefits have been unsatisfactory. ${ }^{2,3}$ In addition, the ACI and MACI cell-based therapies had limitations in that chondrocytes have limited lifespan and both ACI and MACI could not sufficiently correct large cartilage defects. The reason for the failure of these cell-based therapies has been largely because the newly formed tissue as a result of these treatments do not have the structural organization of cartilage and have inferior mechanical properties than the native tissue, and hence often prone to failure. ${ }^{4}$

Various stem cells have been investigated extensively for possible uses in cartilage regeneration, in particular bone marrow mesenchymal stem cells (BMSCs). Whereas there are reports of BMSCs forming cartilage-like tissues in vitro under chondrogenic induction with TGF-beta, once transplanted

\footnotetext{
${ }^{1}$ Institute of Health and Biomedical Innovation, Queensland University of Technology, Brisbane, QLD, Australia; ${ }^{2}$ School \& Hospital of Stomatology, Wuhan University, Wuhan, Hubei, China; ${ }^{3}$ Department of Orthopaedic Surgery, The Second Xiangya Hospital, Changsha, China; ${ }^{4}$ Department of Rheumatism, The Xiangya Hospital, CentralSouth University, Changsha, China and ${ }^{5}$ Prince Charles Hospital, Brisbane, QLD, Australia Correspondence: Dr I Prasadam, PhD or Professor Y Xiao, PhD, Institute of Health and Biomedical Innovation, Queensland University of Technology, 60 Musk Avenue, Kelvin Grove Campus, Brisbane, QLD 4059 Australia. 
into a complex in vivo environment these constructs commonly undergo extensive calcification of the extracellular matrix (ECM) ${ }^{5,6}$ Parathyroid hormone-related protein (PTHrP) has been used to inhibit hypertrophy of BMSCs and subsequent mineralization, $;^{7-10}$ however, there is no evidence to suggest that pre-treating BMSC pellets with PTHrP prevents hypertrophy and mineralization when implanted subcutaneously in nude mice. ${ }^{8}$ The feasibility of using articular cartilage chondrocytes (ACCs) as an alternative source of cells for in vivo transplantations is limited due to the difficulties of obtaining a sufficient number of cells. There is also the issue of ACCs rapidly dedifferentiating when expanded ex vivo which, when implanted in vivo, result in fibrous cartilage. ${ }^{11,12}$ Given these limitations, it is therefore not surprising that cell therapies have failed to gain any widespread adoption as a viable means of treating OA. New approaches are therefore needed to stabilize the phenotypes of both ACCs and BMSCs in order to have any chance of developing a cell-based application for the treatment of OA.

Interestingly, there are now data to suggest that cross-talk between BMSCs and ACCs enhances the expression of cartilaginous ECM components. One in vitro study has shown that ACCs exert paracrine effects on BMSCs by releasing PTHrP, which induces chondrogenesis of BMSCs and inhibits hypertrophy. ${ }^{13}$ Another study seems to suggest that BMSCs can direct ACCs to a more stable phenotype via communication between the two cell types involving the cytokines TGF-beta, BMP-2 and IGF- $1 .{ }^{14}$ A number of other studies have demonstrated that co-culturing BMSCs with ACCs enhances chondrogenesis by increased expression of cartilage ECM components. ${ }^{15-21}$ These observations prompted us to test another strategy for a cell-based OA treatment, namely: to inject into cartilage defects a mixture of BMSCs and ACCs that could provide anti-hypertrophy and pro-chondrogenic cytokines to influence cell differentiation. To date, there are no reports of in vivo studies that have investigated effects derived from combining BMSCs and ACCs to repair cartilage in an OA disease model. Therefore, the aim of this study was to investigate the cellular interactions occurring when mixing ACCs and BMSCs, to determine whether or not such an approach could enhance cartilage regeneration in vitro and ex vivo and also in a medial meniscectomy (MSX) OA model in the rat.

\section{MATERIALS AND METHODS}

\section{Isolation and Culture of Human and Rat BMSCs}

All experiments were conducted in accordance with institutional guidelines for the care and use of experimental animals as stipulated by Queensland University of Technology and the Prince Charles Hospital. Bone marrow samples were obtained from OA patients ( $n=7$; age: $70 \pm 6.3$, sex: males) undergoing knee replacement surgery (KRS) after informed consent had been given. None of these patients had any clinical symptoms of joint, metabolic, or hormonal diseases (osteoporosis). Bone marrow was sourced from the patient's femoral canal and
hBMSCs isolated, cultured, and characterized as described in our previously published studies. ${ }^{22,23}$ Bone marrow from rats was isolated by cutting open both ends of the femurs and flushing out the marrow with a 27-gauge syringe filled with low glucose Dulbecco's modified Eagle's medium (Life Technologies, Australia) containing 1\% (v/v) 50 units $/ \mathrm{ml}$ penicillin and $50 \mu \mathrm{g} / \mathrm{ml}$ streptomycin (pen/strep; Life Technologies) and 10\% FBS (Lonza, Australia) (complete DMEM). The cell suspension was strained through a $70-\mu \mathrm{m}$ mesh filter and cultured in complete DMEM in $25 \mathrm{~cm}^{2}$ flasks in an incubator at $37^{\circ} \mathrm{C}$ and $5 \% \mathrm{CO}_{2}$. Cells were passaged once reaching $80-90 \%$ confluence and rBMSCs were confirmed by their differentiation potential and detection of MSCs surface markers expression by FACS and RT-qPCR as described previously. ${ }^{22,23}$

\section{Isolation and Culture of Human and Rat ACCs}

Human ACCs (hACCs) were obtained from the non-loadbearing mid-region of lateral compartment of the knee, from the same KRS patient cohort $(n=7)$. The isolation protocol of both human and rat (h and $\mathrm{r}$ ) ACCs was essentially the same and has been described previously. ${ }^{24,25}$ Briefly, articular cartilage was removed from the femoral condyles. The tissue was washed three times with sterile $1 \times$ PBS supplemented with $1 \%(\mathrm{v} / \mathrm{v})$ pen/strep, then cut into $1-2 \mathrm{~mm}^{3}$ pieces and digested in a solution $0.2 \%(\mathrm{v} / \mathrm{v})$ collagenase B (Life Technologies) in complete DMEM for $24 \mathrm{~h}$ in a $\mathrm{CO}_{2}$ incubator. The digested cartilage was centrifuged at $400 \mathrm{~g}$ for $10 \mathrm{~min}$ and the supernatants discarded, after which the cells were strained through a $70-\mu \mathrm{m}$ mesh filter and cultured in complete DMEM. Phenotype characterization of the ACCs were assessed by the cells' ability to undergo chondrogenic redifferentiation and the expression of type I collagen (COL1), type II collagen (COL2) (Neomarkers, Fremont, CA, USA) and aggrecan (ACAN) (Millipore, Australia) as described previously. ${ }^{24,25}$

\section{In Vitro Co-Culture Model}

ACCs $\left(1.5 \times 10^{5}\right)$ from primary cultures and BMSCs $\left(1.5 \times 10^{5}\right.$ cells) from passage 1 were combined (1:1 BMSCs/ ACCs). The co-cultures were established in a micromass culture system by pelletizing the cells in a chondrogenic medium comprising serum-free high-glucose DMEM (Life Technologies) supplemented with $10 \mathrm{ng} / \mathrm{ml}$ TGF- $\beta 3$ (R\&D Biosystems), $10 \mathrm{~nm}$ dexamethasone (Sigma-Aldrich), $50 \mathrm{mg} /$ $\mathrm{ml}$ L-ascorbic acid (Sigma-Aldrich), $10 \mathrm{mg} / \mathrm{ml}$ sodium pyruvate (Life Technologies), $10 \mathrm{mg} / \mathrm{ml}$ proline (SigmaAldrich), and ITS+ (final concentration: $6.25 \mathrm{mg} / \mathrm{ml}$ insulin, $6.25 \mathrm{mg} / \mathrm{ml}$ transferrin, $6.25 \mathrm{mg} / \mathrm{ml}$ selenious) acid (Life Technologies), $5.33 \mathrm{mg} / \mathrm{ml}$ linoleic acid (Sigma-Aldrich), and $1.25 \mathrm{mg} / \mathrm{ml}$ bovine serum albumin (Sigma-Aldrich) and centrifuged at $600 \mathrm{~g}$ for $20 \mathrm{~min}$ to form a pellet. Controls were pellets made from the same number of BMSCs or ACCs only, also cultivated in chondrogenic medium. 


\section{In Vivo Cartilage Defect Model}

The ex vivo cartilage defect model was prepared from cartilage harvested from the KRS patients' excised knee joints. Only cartilage from the mid-region of the lateral-femoral compartment, showing no visual damage, was used in this experiment. Six millimeter diameter with $3 \mathrm{~mm}$ depth cartilage was dissected using a sharp biopsy punch and circular defects of $2 \mathrm{~mm}$ in diameter and $2 \mathrm{~mm}$ deep, were made using a dental bur and the site was irrigated with generous amounts of PBS to prevent overheating. After having been thoroughly washed with PBS the cartilage defects received pellet implants containing hACCs, human BMSCs (hBMSCs), or mixed (hBMSCs+hACCs) that had been incubated in chondrogenic medium for 3 weeks. The defects were sealed with matrigel (Figure 1a and b) and then transplanted subcutaneously into non-obese diabetic severe combined immunodeficient mice (NOD-SCID; Animal Resource Center, WA, Australia) for a further 3 weeks. Cartilage defect explants filled with matrigel only or cartilage plug itself without gel served as controls. This procedures had the approval of the Animal Ethics Committee of the Queensland University of Technology.

\section{In Vivo Meniscectomy-Induced OA Model and Intra- Articular Stem Cell Injections}

Post-trauma OA was induced by MSX surgery as described in our previous study. ${ }^{26}$ In brief, Wistar rats were anesthetized with an intra-peritoneal injection of Zoletil (tiletamine $15 \mathrm{mg} /$ $\mathrm{kg}$, zolazepam $15 \mathrm{mg} / \mathrm{kg}$ ) and Xylazil (xylazine $10 \mathrm{mg} / \mathrm{kg}$ ) and $\mathrm{OA}$ induced by transecting the medial collateral ligament just below its attachment to the meniscus, which causes the meniscus to be reflected toward the femur when the joint space is opened. Avoiding damage to the tibial surface, the meniscus was cut at its narrowest point, which results in complete medial meniscus transection, and the surgical wound was closed by suturing in two layers. A group of sham-operated animals had their left knee subjected to the same surgical procedure but without excision of the ligament or any meniscal manipulations. After surgery, the rats all received pain killer (Buprenorphine $0.05 \mathrm{mg} / \mathrm{kg}$ ) and antibiotic (gentamycin $5 \mathrm{mg} / \mathrm{kg}$ ). One week post-surgery, the rats received a designated single $75 \mu \mathrm{l}$ dose of either rBMSCs $\left(3 \times 10^{5}\right.$ cells $)$, rACCs $\left(3 \times 10^{5}\right.$ cells $)$, or mixed rBMSCs $+\mathrm{rACCs}\left(1.5 \times 10^{5}\right.$ BMSCs+1.5 $\times 10^{5}$ ACCs $)$ dissolved in complete DMEM supplemented with hyaluronic acid (HA) gel $(0.5 \mathrm{mg})$ injected directly into the knee joint. The same volume of complete DMEM and HA only was injected into the knees of control animals. Following an injection, the joint was repeatedly flexed and extended to help disperse the suspension throughout the intra-articular space, after which the rats were allowed to walk freely in the cage. The animals were killed 10 weeks post-surgery by $\mathrm{CO}_{2}$ inhalation.
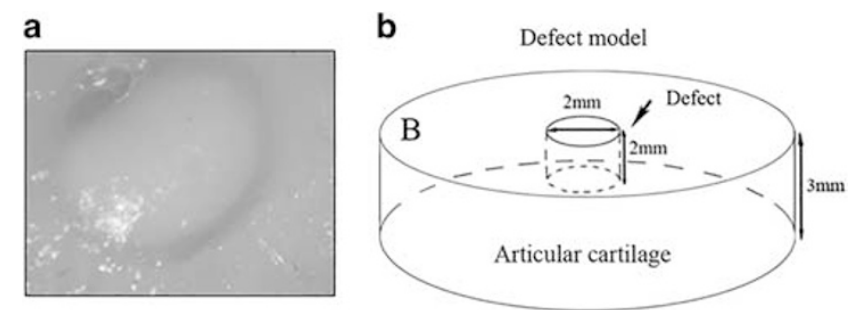

Figure 1 Ex vivo cartilage defect model. (a) Pellet of mixed ACCs + BMSCs at week 3 about to be transplanted into an ex vivo cartilage defect and sealed with matrigel and implanted subcutaneously in SCID mice as described in the Materials and methods section (right panel). (b) Schematic representation of the cartilage defect model.

\section{MTT Assay}

Cell proliferation was assessed using (3-(4,5-dimethylthiazol2-yl)-2,5-diphenyltetrazolium bromide (MTT; SigmaAldrich) assay. Twenty microliters of $5 \mathrm{mg} / \mathrm{ml}$ MTT solution was added to each well and incubated for $4 \mathrm{~h}$ at $37^{\circ} \mathrm{C}$, after which the medium was carefully removed and replaced with $100 \mu \mathrm{l}$ of dimethyl sulfoxide to dissolve the formazan crystals. The absorbance was read at $570 \mathrm{~nm}$ by a microplate spectrophotometer (Benchmark Plus, Tacoma, WA, USA).

\section{Sulfated Glycosaminoglycan Assay}

Sulfated glycosaminoglycan (sGAG) assay was performed using a Sulphated Glycosaminoglycan Assay kit (Biocolor Life Science Assays, Labtek Pty, QLD, Australia) following the manufacturer's instructions. Chondrocytes pellets were digested with a papain extraction reagent overnight at $65^{\circ} \mathrm{C}$ to recover sGAGs and mixed with blyscan dye. The color intensity was measured at $656 \mathrm{~nm}$ on a Benchmark Plus microplate spectrophotometer (BioRad, Australia).

\section{RNA Extraction and Real-Time Quantitative PCR}

Total RNA was extracted from pellet cultures using TRIzol reagent (Life Technologies) as described in our previous studies. ${ }^{24,25}$ Complementary DNA was transcribed from $500 \mathrm{ng}$ of mRNA using a DyNAmo kit (Thermo Scientific). Real-time quantitative PCR (RT-qPCR) was performed using SYBR Green master mix on a ABI 7500 Fast Real Time PCR system (Applied Biosystems, Foster City, CA, USA). All samples were assayed in triplicate and the mean cycle threshold $(\mathrm{Ct})$ values calculated for comparative analysis using the $\left(2^{-\Delta \mathrm{Ct}}\right) \operatorname{method}^{27,28}$ and $18 \mathrm{~s}$ and beta-actin expression was used as the internal controls. Geometric mean of $18 \mathrm{~s}$ and beta-actin housekeeping genes are used for relative gene expression analysis.

\section{Safranin-O Staining}

In vitro pellets and dissected explants were fixed in $4 \%$ paraformaldehyde (PFA) and embedded in paraffin. Whole knee joints were first fixed in PFA and then decalcified in 10\% EDTA for 5-6 weeks before embedding in paraffin. Sagittal 
a

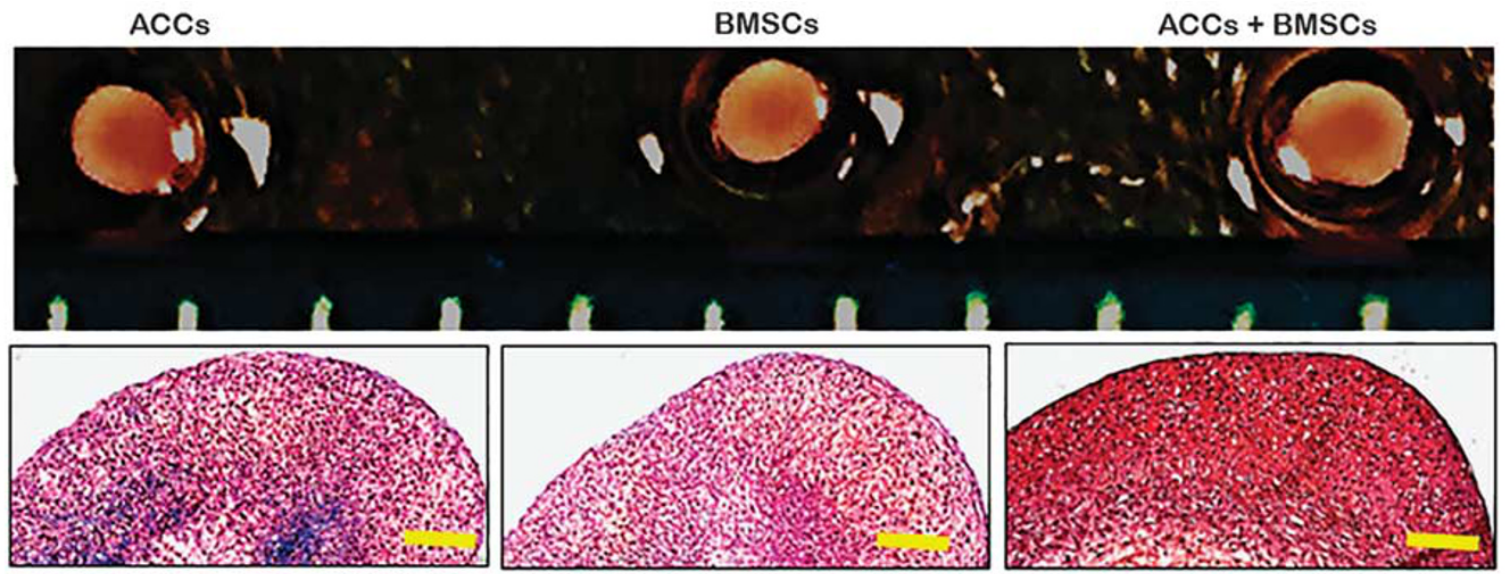

b

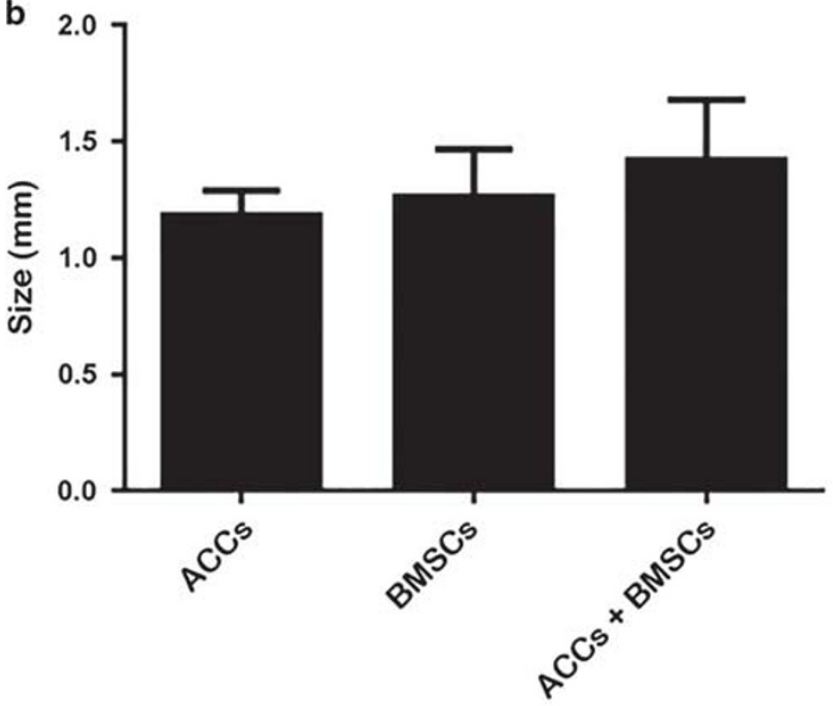

d

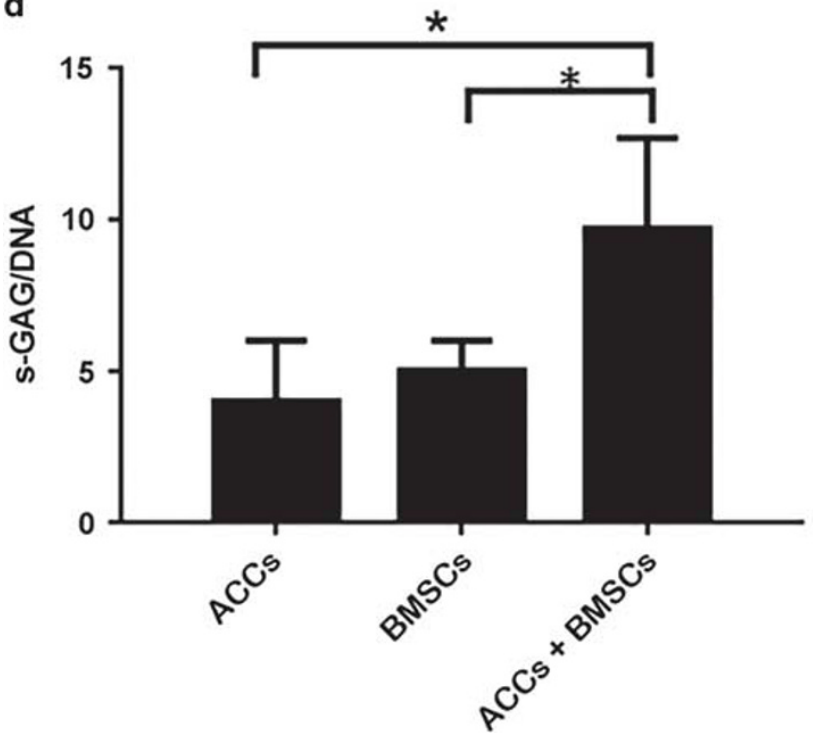

c

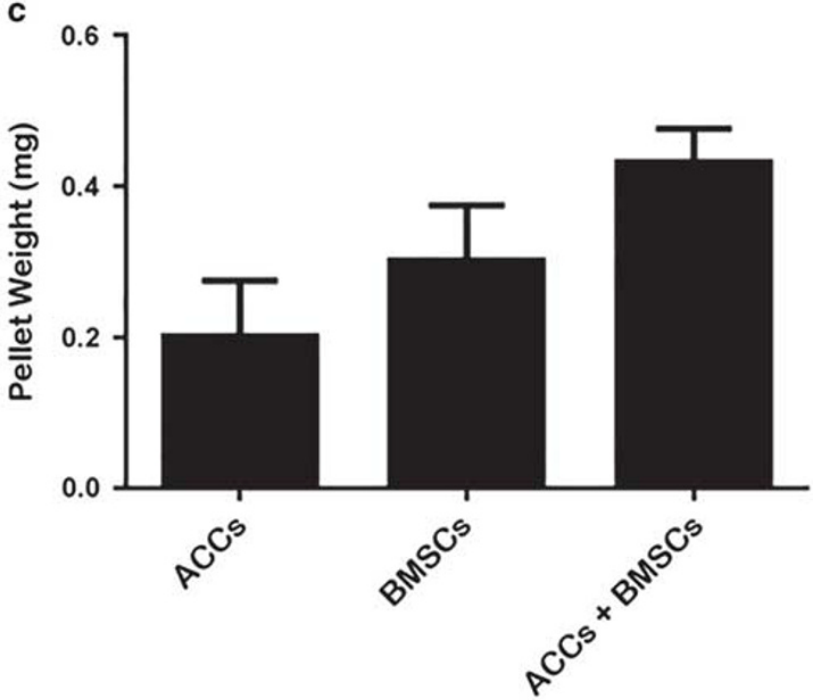

e

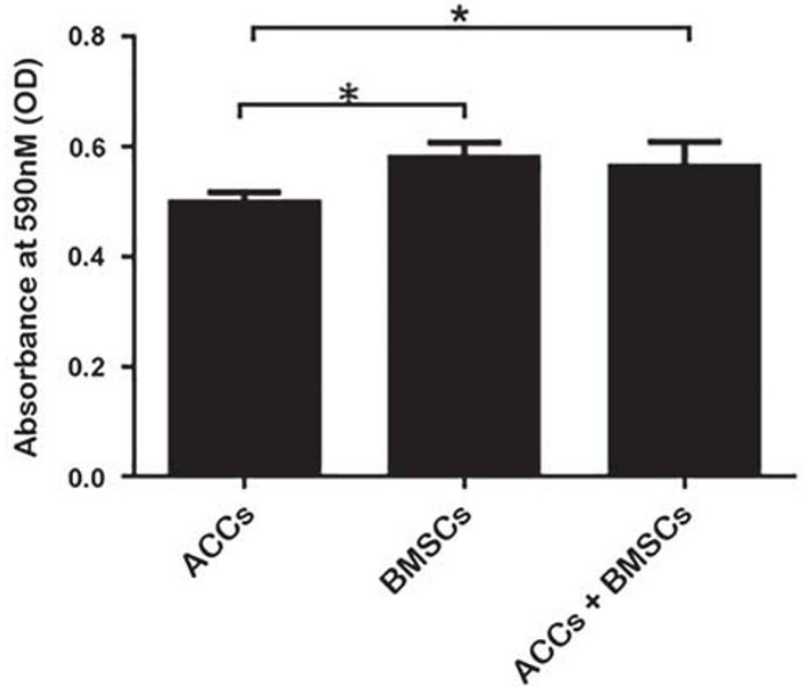

Figure 2 Enhanced in vitro chondrogenic differentiation in the co-culture of human BMSCs+ACCs. BMSCs only, ACCs only, or BMSCs+ACCs (1:1) were differentiated in chondrogenic induction medium for 3 weeks and analyzed by (a) gross morphology and safranin-O staining analysis of chondrogenic differentiated BMSCs, ACCs, and ACCs+BMSCs pellets; (b) chondrocyte pellet size; (c) pellet wet weight; (d) total sGAG/DNA content; and (e) MTT cell proliferation assay. ${ }^{*} P<0.05$, results are representative of studies performed in three different patient samples with two technical replicates. Data are reported as means \pm s.d.; $* P<0.05$. 
sections, $5 \mu \mathrm{m}$ in thickness, were cut from the lateral and medial compartment of the joint. Matrix deposition of proteoglycans was assessed using Safranin-O (Saf-O) staining following the standard protocols ${ }^{29}$ Bern score was used to analyze the Saf-O results ${ }^{30}$ and OA severity in the tibial plateau was evaluated according to Mankin's histologic grading system by three blind scorers (Mankin score: 0 to 14) ${ }^{31}$ and a cartilage destruction score was assigned to each knee sample.

\section{Immunohistochemical Analysis}

Immunohistochemistry was carried out using standard protocols for paraffin-embedded samples. Sections were incubated with primary antibody overnight at $4{ }^{\circ} \mathrm{C}$ and the following primary antibodies and dilutions were used: COL10 (Santa Cruz Biotechnology, 1:500); ACAN (Abcam, 1:50); MMP-13 (Labvison, 1:500); ChM-1 (Santa Cruz Biotechnology, 1:100); COL2 (Abcam, 1:200); COL1 (Abcam, 1:300), and VEGF (Neomarkers, 1:500). The following day the sections were incubated with biotinylated swine-anti-mouse, rabbit, goat secondary antibody (Dako Multilink) for $40 \mathrm{~min}$ and the antibody complexes visualized using a diaminobenzidine (DAB) substrate and counterstained with Mayer's hematoxylin. Controls for the immunostaining procedures included conditions where the primary antibody or the secondary (anti-mouse IgG) antibodies were omitted. Images were captured on a Leica SCN400 slide scanner (Leica Biosystems, Australia).

\section{Statistical Analysis}

Results were expressed as means \pm s.d. Comparison of gene expression values between two groups was performed using unpaired $t$-test or the nonparametric Mann-Whitney test and multiple comparisons were performed with Kruskal-Wallis and Dunn's post hoc tests. $P$-values of $(P \leq 0.05)$ were considered significant. Graph Prism is used for statistical analysis and graphical representation.

\section{RESULTS \\ Enhanced In Vitro Chondrogenic Differentiation in the Co-Culture of hBMSCs and hACCs}

The gross morphology of the pellets was identical in either mixed or monocultures (Figure 2a). After 3 weeks in culture there was no statistical difference in the size or weight of the pellet aggregates, with the BMSCs+ACCs mixed pellets being only slightly larger (Figure $2 \mathrm{~b}$ and $\mathrm{c}$ ). Next, we examined in vitro chondrogenic differentiation in mixed and monocultures of hBMSCs and hACCs. All pellets stained positive for Saf-O, indicating the formation of ECM; however, the staining intensity was higher in mixed BMSC+ACC pellets compared with the BMSC and ACC pellets alone. In the mixed pellets the staining was an intense red, covering more than $90 \%$ of the total tissue area stained. By contrast, the Saf$\mathrm{O}$ stain in the ACC pellets was more diffuse and in a spotty pattern, covering only $63 \%$ of the stained area. The matrix staining in BMSC pellets looks similar to ACCs (Figure 2a). Sulfated GAG is the major metrics with which to assess chondrogenic differentiation and agreed with the Saf-O staining, with total sGAG being significantly higher in the mixed cultures compared with the monocultures (Figure 2d).

The rate of proliferation was similar between BMSCs monocultures and BMSCs+ACCs mixed cultures, whereas the ACCs monocultures were lower by comparison (Figure 2e).

Chondrogenic gene expression was assessed by RT-qPCR and showed significantly higher levels of COL2A1, ACAN, and SOX9 mRNA in the mixed BMSC+ACC pellets compared with the monoculture pellets. The BMSCs-only pellets had higher expression of the hypertrophy and mineralization marker genes COL10, RUNX2, MMP-13, and ALPL compared with the ACC monocultures. The mixed culture BMSC+ACC showed lower expression of these markers compared with the other groups. The expression of the hypertrophic transcription factor $I H H^{32}$ was similar in both monocultures but significantly lower in mixed culture pellets. Interestingly, the angiogenic gene $V E G F$ had an inverse expression pattern compared with the anti-angiogenic gene ChM-1. VEGF expression in the BMSC group was significantly higher compared with the BMSC+ACC group, whereas ChM-1 expression was upregulated in the $\mathrm{BMSC}+\mathrm{ACC}$ group compared with the monoculture groups (Figure 3). In terms of gene expression, these observations indicate that the BMSCs+ACCs mixed cultures had a more stable cartilage-like phenotype.

\section{Co-Culture of ACCs+BMSCs Showed Phenotypic Stability After Subcutaneous Implantation in SCID Mice} Gross and histological findings in cartilage defect explants

The cartilage implants were harvested 3 weeks after implantation and all had remained within their original subcutaneous pockets dorsally in the SCID mice. The cartilage defects filled with ACC-derived pellet or mixed cell pellets had a smooth and white surface, showing no signs of blood vessel invasion (Figure 4a). By contrast, cartilage defects filled with BMSC-derived pellets or Matrigel only showed evidence of blood vessels invasion (Figure 4a).

\section{Evaluation of cartilage explants by safranin-O staining}

Saf-O staining was used to assess proteoglycan production, being a measure of chondrogenesis within the cartilage defects after 3 weeks in a subcutaneous pocket. This revealed a surprisingly intense staining within the cartilage defect explants treated with the BMSCs+ACCs cell pellets which were equal to that of the cartilage. By contrast, the ACCs treatment exhibited a lighter, pink staining of the implanted pellets, whereas the BMSC-treated defects showed no Saf-O within the cell pellet (Figure 4b). Microscopic analysis revealed the presence of chondrocyte-like cells integrated at the boundary with the native cartilage in ACCs-derived pellets and mixed cell-derived pellets (Figure 4b). This was not observed in the BMSCs-derived pellets, in which only 

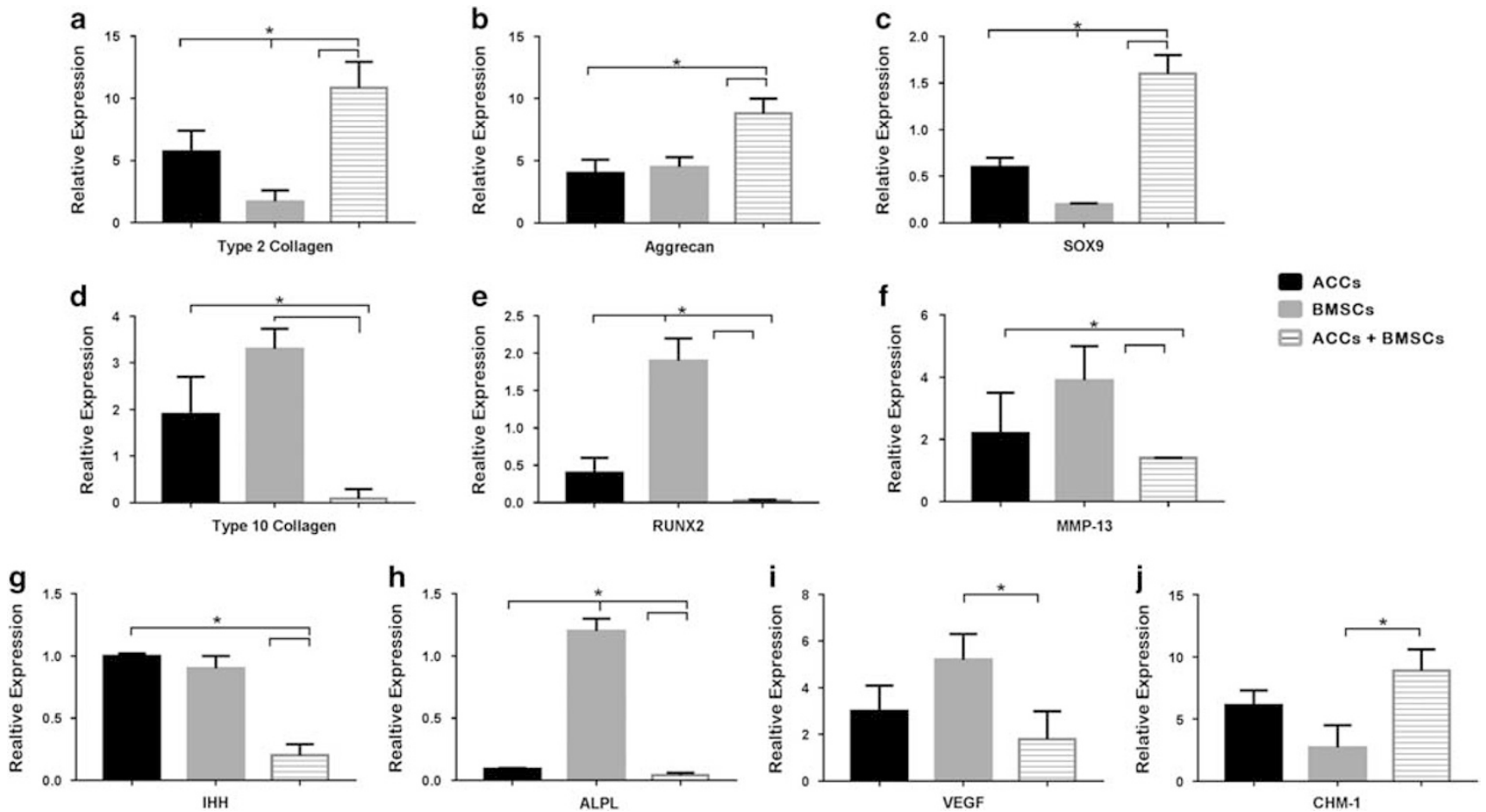

Figure 3 Chondrogenic, hypertrophic, angiogenic, and anti-angiogenic gene expression patterns in the co-culture vs non co-cultured pellets. ACCs only, BMSCs only, or BMSCs+ACCs (1:1) were differentiated in chondrogenic induction medium for 3 weeks and mRNA expression levels quantified by RTqPCR. There was significantly higher gene expression of the chondrogenic markers COL2, ACAN, and SOX9 and the anti-angiogenic marker ChM-1 in the BMSC+ACC co-culture groups compared with the monoculture groups. By contrast, the gene expression of hypertrophic markers COL10, RUNX2, MMP-13, IHH, and ALPL and the angiogenic factor VEGF were lower in the mixed ACCs+BMSCs groups compared with the BMSCs alone group. The results are representative of experiments using three different patient samples with three technical replicates. Data are reported as means \pm s.d.; ${ }^{*} P<0.05$.

fibroblast-like cells surrounded the defect site (Figure $4 \mathrm{~b}$ ). In the Matrigel-only controls, there was no evidence of tissue formation when compared with the cell treatment groups. Bern score analysis showed a significant upregulation in cartilage defects treated with ACC+BMSC pellets compared with the ACCs- and BMSCs-only pellets $(P<0.05)$ (Figure 4c).

\section{Immunohistochemical staining}

The cartilage defects were assessed by immunohistochemistry using a panel of antibodies covering the expression of chondrogenic marker (ACAN and COL2), angiogenesis marker (VEGF), angiogenesis inhibitor (ChM-1), hypertrophic markers (COL10), degradative marker (MMP-13), and de-differentiation (COL1) marker. Both ACAN and COL2, and ChM-1 were highly expressed in the mixed BMSCs+ACCs group compared with the ACCs- and BMSCsonly pellets (Figure $5 \mathrm{a}$ and $\mathrm{b}$ ). The expression of COL10 and MMP-13 and VEGF in the ACCs+BMSCs group stood out as being significantly lower compared with the BMSCs-only group and this expression pattern mirrored with ACCs-only group. The expression of COL1 remains similar in all groups (Figure $5 \mathrm{a}$ and $\mathrm{b}$ ).
Intra-articular injections of mixed ACCS+BMSCs cells promoted cartilage repair in MSX-induced osteoarthritis rat model compared with monoculture cell treatments

The effects of the three cell configurations on the repair of articular cartilage damaged by MSX-induced OA in the rat was assessed by histochemical analysis. After 10 weeks in situ, the cartilage in the MSX models showed surface irregularities, abrasion, total matrix loss, and chondrocyte disappearance, all typical characteristics of human OA cartilage (Figure 6). The changes were evaluated quantitatively using the Mankin scoring system and this showed a significantly higher Mankin score in the MSX group compared with the sham-operated group (Figure 7a). Saf-O staining revealed that the mixed BMSCs+ACCs treatment group showed significantly more intense cartilage matrix staining and, therefore, better cartilage repair compared with the ACCs alone and BMSCs alone (Figure 6), finding that were reflected in the Mankin scores (Figure 7a).

\section{DISCUSSION}

The use of cell-based therapies to regenerate defective cartilage has been the focus of numerous animal studies over the past four decades, as well as clinical trials in humansfirst pioneered by the Swedish surgeon Brittberg in the early 


\section{b}
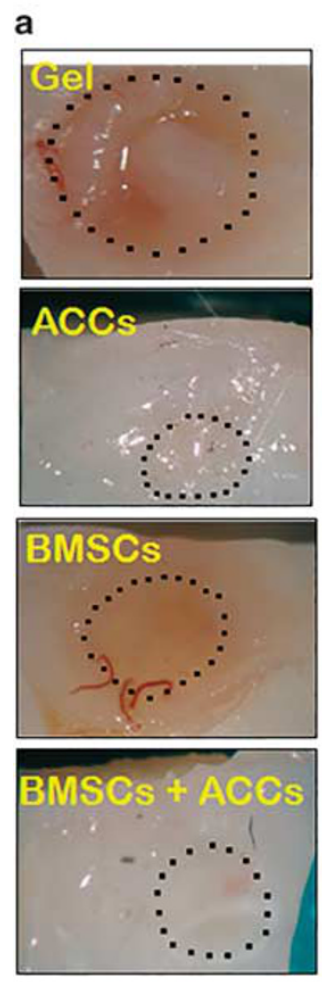
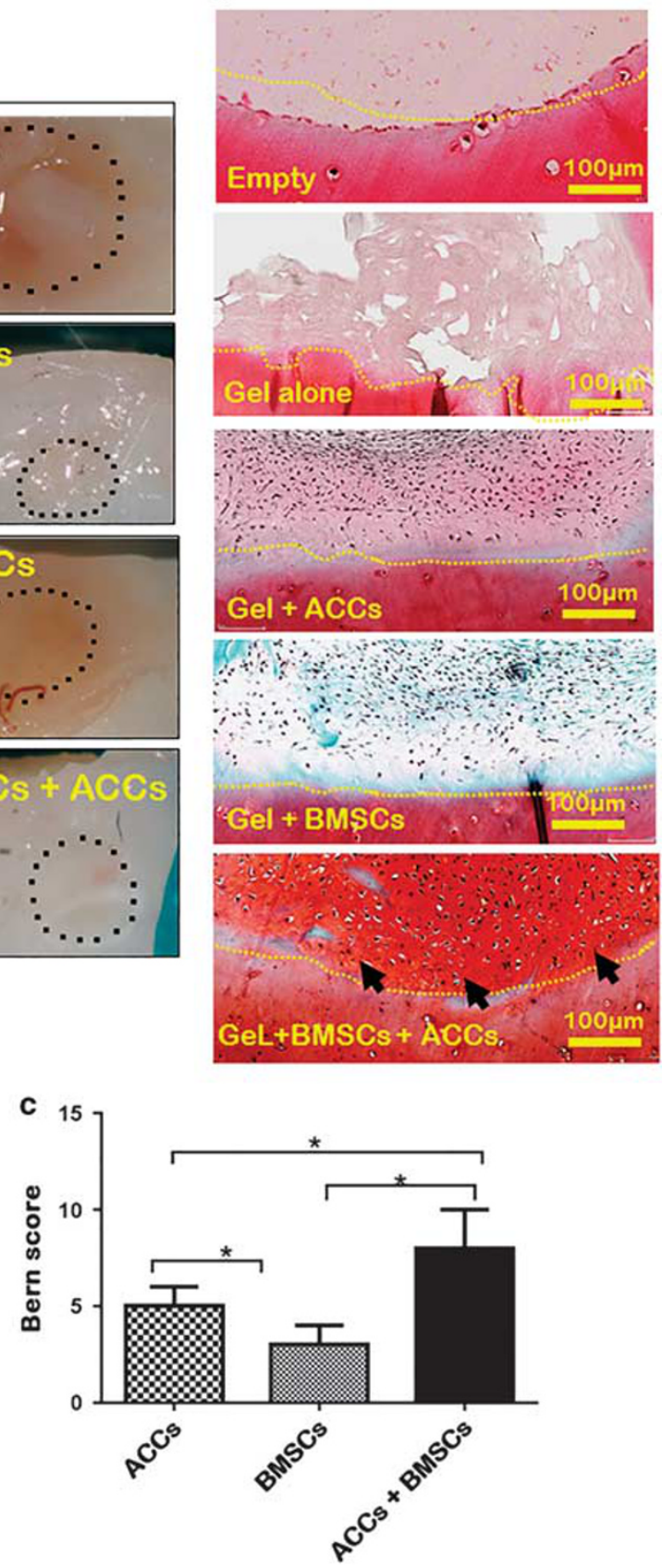

1990s. ${ }^{33}$ To date, however, this method has failed to meet expectations as a panacea for cartilage repair. The key obstacle is the limited supply of autologous ACCs that can realistically be harvested from a patient without causing further damage to the articular cartilage adjacent to the site of explanation. From the small pool of ACCs that can be isolated from the patient's own cartilage, it is necessary to first expand the cells ex vivo over many population doublings in order to obtain a sufficiently high number to use for cartilage regeneration. ${ }^{34}$ Chondrocytes are known to dedifferentiate after $2-4$ passages
Figure 4 Co-culture of hBMSCs and hACCs improved cartilage formation in an ex vivo cartilage defect model. Cell pellets (ACCs, BMSCs, and ACCs +BMSCs) were seeded into an ex vivo cartilage defect after 3 weeks of chondrogenic induction and sealed in matrigel before being implanted subcutaneously in SCID mice for a further 3 weeks. (a) After 3 weeks both the ACCs-only pellets and mixed cell pellets can be seen underneath a smooth, white surface, showing no signs of blood vessels invasion. By contrast, with the BMSCs-only pellets and empty controls there was clear evidence of blood vessel formation. Representative images are shown from experiments from three pellets per group, sourced from three different patients; scale bar $=50 \mu \mathrm{m}$. (b) Safranin-O staining was used to assess ECM proteoglycan deposition following 3 weeks subcutatneous implantation in SCID mice. There was significantly more intense staining in the mixed ACCs+BMSCs group, followed by the ACCs group compared with the BMSCs and empty control group. (c) Bern scores of cartilage formation in ACCs $(n=3)$, BMSCs $(n=3)$, and ACCs+BMSCs groups $(n=3)$. Results are representative of studies performed on three different patient samples with three technical replicates. Data are reported as means \pm s.d.; ${ }^{*} P<0.05$.

when expanded in $2 \mathrm{D}$ cultures ${ }^{35-37}$ and, furthermore, when implanted in vivo, ACCs will typically form fibrous cartilage rather than the hyaline form that is characteristic of articular cartilage. ${ }^{34}$ Given the short supply of ACCs, there have been concerted efforts to use BMSCs as a potential source of cells for cartilage regeneration. However, it has been shown that BMSCs, when implanted in vivo, express cartilage hypertrophic markers such as COL10, FGF receptors 1-3, PTHrP, and MMP-13 and also exhibit increased ALP activity and matrix mineralization, resulting in local calcification and blood vessel invasion. ${ }^{38}$ Similar expression of hypertrophic markers has also been observed in stem cells such as adiposederived mesenchymal stem cells. ${ }^{39}$ These observations highlight the need for strategies to minimize these drawbacks in order to successfully apply cell-based treatments to repair articular cartilage lesions.

While other studies have tested the in vitro chondrogenic potential of combining ACCs and BMSCs, ${ }^{14,40}$ the present study is the first of its kind to provide in vivo data that demonstrate the efficacy and enhanced chondrogenic potential of mixed ACCs+BMSCs cultures in an ex vivo cartilage defect model implanted subcutaneously in immune deficient mice, as well as injected directly into the knees of rats with MSX-induced OA, thus providing proof-of-concept that this strategy could be implemented for cartilage regeneration in OA patients.

Our study revealed that co-cultures containing BMSCs and ACCs robustly enhanced in vitro chondrogenesis while suppressing hypertrophy. The co-cultures also upregulated anti-angiogenesis-associated genes and, at the same time, inhibited the expression of angiogenic factors, indicating that the communication between the two types of cells can lead to more stable chondrogenic gene expression, observations that were consistent with previously published in vitro studies. For example, it has been reported that mixing ACCs with BMSCs enhances the expression of the chondrogenic genes COL2 and 

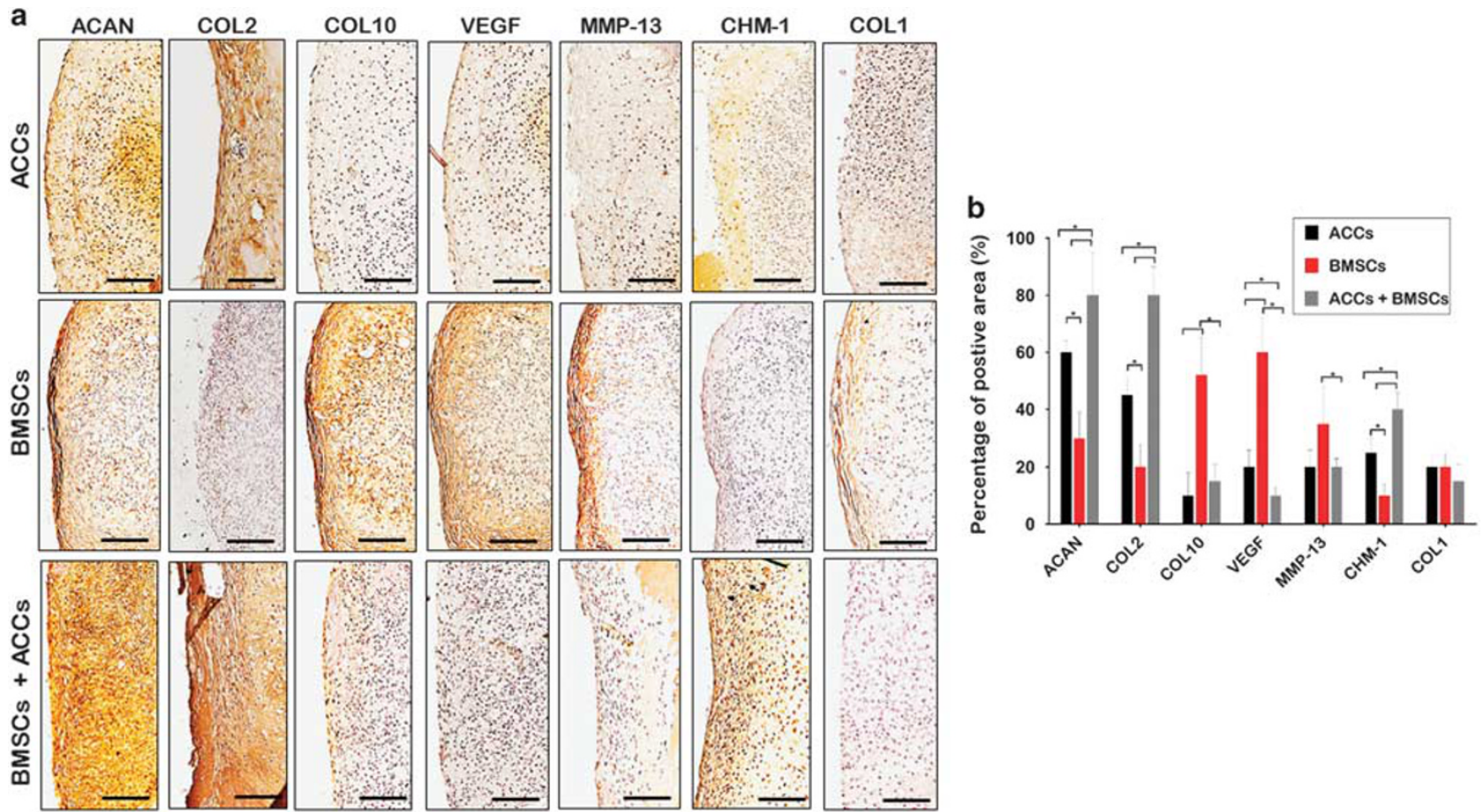

Figure 5 Co-culture of hBMSCs and hACCs enhances cartilage phenotype in an ex vivo cartilage defect model. Following 3 weeks of growth in chondrogenic induction medium, cell pellets (ACCs, BMSCs, and ACCs+BMSCs) were placed in cartilage defect explants and sealed with matrigel before being implanted subcutaneously in SCID mice for further 3 weeks. (a) IHC staining was performed to assess expression of chondrogenic marker (ACAN and COL2), hypertrophic markers and degradative (COL10 and MMP-13) and angiogenesis factor (VEGF) and angiogenesis inhibitor (ChM-1) and dedifferentiation marker (Col1). (b) Quantification of staining in the mixed ACCs+BMSCs group compared with the ACCs and BMSCs-only groups. Data are reported as means \pm s.d.; ${ }^{*} P<0.05$. Scale bar $=50 \mu \mathrm{m}$. Results are representative of studies performed on three different patient samples with two technical replicates.

ACAN.${ }^{15}$ Similarly, co-cultures of BMSCs and ACCs enhance chondrogenesis while suppressing hypertrophy in vitro. ${ }^{40-42}$ The role of VEGF in angiogenesis driven blood vessel formation in OA is well known ${ }^{43,44}$ and it is firmly established that VEGF have pathological role in OA cartilage. ${ }^{45} \mathrm{ChM}-1$, on the other hand, inhibits angiogenesis in cartilage ${ }^{46,47}$ and its high expression by chondrocytes helps to maintain their avascular phenotype. Our findings indicate that BMSCs, even in chondrogenic culture conditions, will naturally express higher levels of VEGF and lower levels of ChM-1; ACCs, by contrast, express higher levels of ChM-1 and lower levels of VEGF under the same culture conditions. However, when the two cell types were mixed together the expression of VEGF was suppressed and ChM-1 was upregulated to a higher level compared with monocultured cells, suggesting that communication between ACCs and BMSCs actively supresses cartilage vascularization, a mechanism that has yet to be unravelled. We made similar observations in the ex vivo cartilage defect model. Whereas there was clear evidence of blood vessel formation in BMSC-treated cartilage defects implanted subcutaneously in the SCID mice, we found no evidence of vascularization in cartilage defects treated with mixed ACCs+BMSCs pellets, further indicating that cocultures exerted an inhibitory effect on angiogenesis.
The effectiveness of BMSCs and ACCs mixed cell in augmenting cartilage regeneration and OA has not previously been tested in vivo, prompting us to test whether the results seen with mixed cell cultures in vitro could be mimicked in animal models. We applied an MSX-induced OA model in rats, a model which we have previously reported closely mimics human OA. ${ }^{26}$ An advantage of this particular model is that, unlike cartilage defect models, the underlying bone is not penetrated during $\mathrm{OA}$ induction, which minimizes the possibility of bone marrow progenitors entering into the defect site and contributing to cartilage regeneration. ${ }^{48}$ Therefore, one can reasonably assume that any evidence of regeneration in the induced cartilage lesion is the result of the injected cells delivered via the intra-articular route. Interestingly, we found that when BMSCs or ACCs only were injected intra-articularly in the MSX rats the cells attached to the injured cartilage surface and formed a layer on top of the existing cartilage, a process that appears to contribute to the repair. This contrasted with the mixed cultures which did not exhibit this effect, although the cell density was higher compared with the sham-operated controls in the given amount of time. By the end of the 10-week treatment period, the mixed BMSCs+ACCs treatment showed significantly higher proteoglycan production and cartilage matrix deposition compared with either ACCs or BMSCs treatments, 

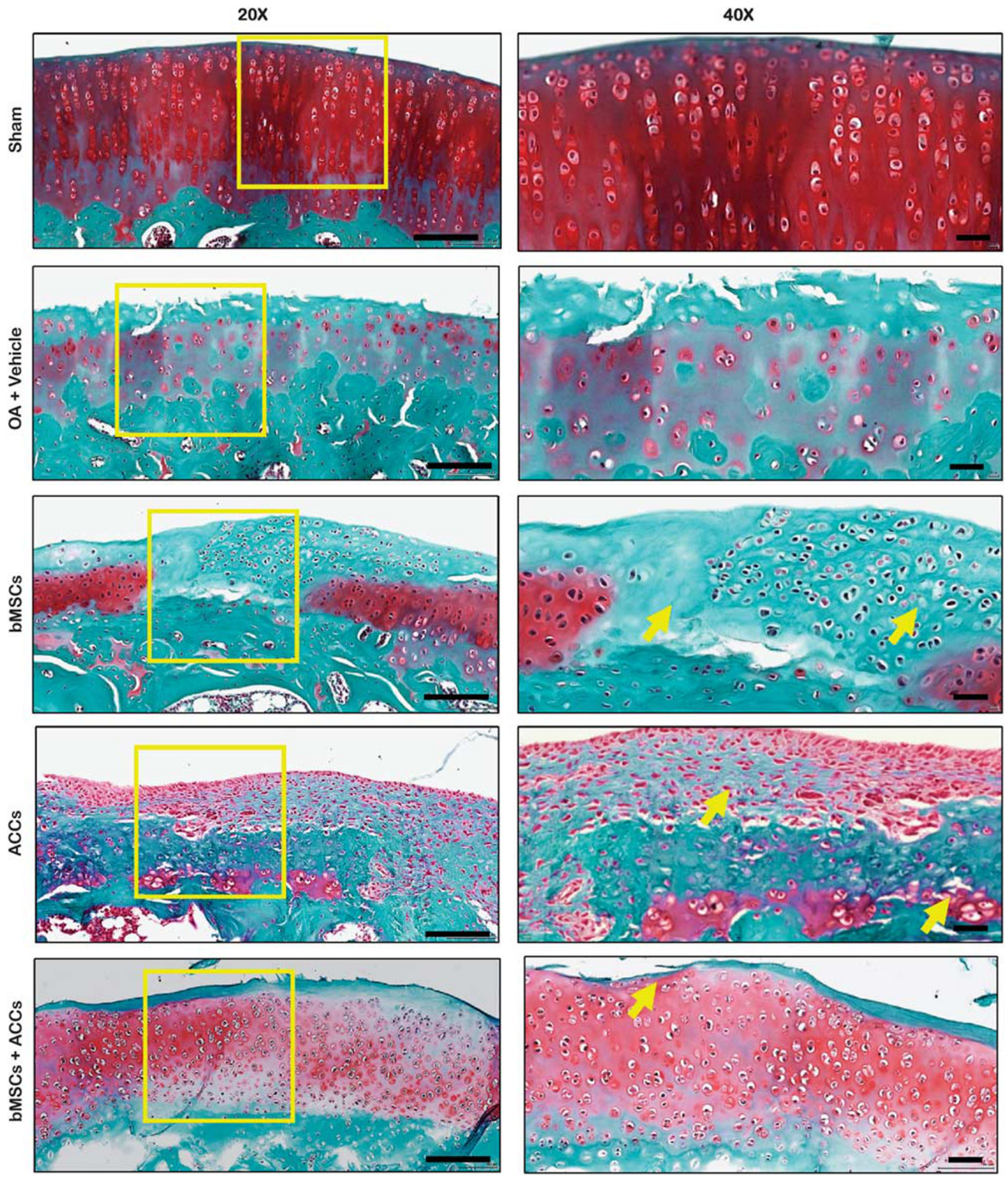

Figure 6 Intra-articular injection of mixed ACCs+BMSCs cell therapy showed enhanced cartilage repair in the MSX rat OA model. OA was induced in rats by meniscectomy surgery. Undifferentiated cells of either BMSCs $\left(3 \times 10^{5}\right.$ cells; $\left.n=7\right)$ or ACCs $\left(3 \times 10^{5}\right.$ cells; $\left.n=7\right)$ or BMSCs+ACCs $\left(1.5 \times 10^{5}\right.$ BMSCs $+1.5 \times 10^{5}$ ACCs; $n=7$ ) were injected intra-articularly into the right knee joint of the rats 1 week post-surgery. The animals were killed 10 weeks after receiving the single injection of cells. Safranin-O staining was used to assess proteoglycan deposition and cartilage matrix formation in the tibia focusing on load-bearing medial compartment. The ACCs+BMSCs treatment group showed enhanced ECM matrix deposition in the OA rat model. The scale bars represents $200 \mu \mathrm{m}$. Images are representative of experiments performed on seven different animals. Yellow squares represent the magnified areas and arrows indicated the regenerated tissue. 


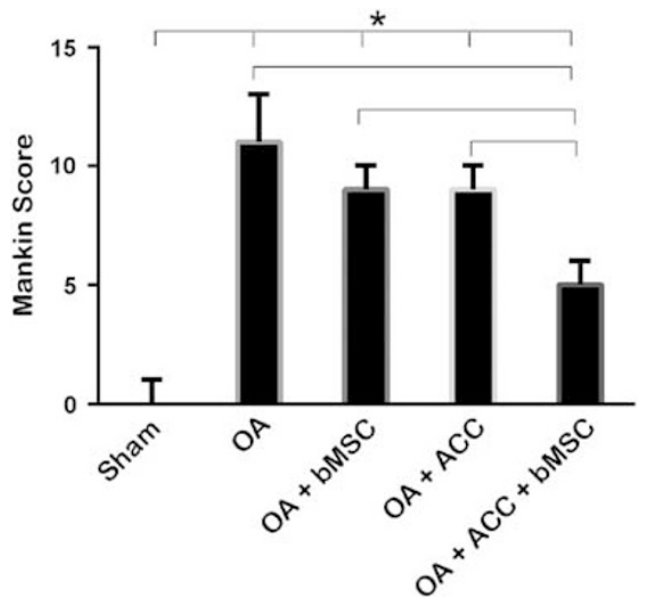

Figure 7 Cell-based treatment with mixed ACCs+BMSCs attenuated the severity of OA cartilage degeneration in MSX rat OA model. (a) Mankin grading of cartilage lesions showed significantly lower score in the ACCs +BMSCs treatment group compared with ACCs or BMSCs-only treatments or control OA-only groups. $n=7$. Data are expressed as mean \pm s.d. Graphs are representative of experiments performed on seven animals. ${ }^{*} P<0.05$.

suggesting that the combination of the two had an effect on the regeneration of damaged cartilage.

The implication of these findings is the need to identify the factors in the co-cultures that induce the chondrogenic phenotype. Questions such as what signaling pathways are involved, whether the cellular communication is bi-directional and if it acts via direct or indirect cell communication need to be addressed. Indirect cell communication would take place by means of soluble factors such as cytokines and growth factors. For example, ACCs secrete factors such as TGF- $\beta^{49}$ and bone morphogenetic proteins, ${ }^{50}$ which have been shown to promote in vitro chondrogenesis of BMSCs. Interleukin-1 beta (IL-1 $\beta$ ), IL-6, and IL-8 are other signaling molecules that have been found at significantly higher levels in the supernatants of BMSC and ACC co-cultures. ${ }^{51}$ Another potential mode of action could be direct cross-talk between cells acting via gap junctions and integrin receptors. Moreover, cells are known to secrete a large variety of vesicles into the extracellular space, of which exosomes have received the most attention in recent years. They were originally thought to be necessary for the clearance of unneeded proteins from cells, and the current opinion is that exosomes are specifically secreted vesicles involved in intercellular communication. Because exosomes are involved in cell-to-cell communication, it is possible they are paracrine effectors of MSCs and ACCs. Our current data, however, do not answer whether the host tissue signals or injected signals in ACCs+BMSCs contributed to the tissue regeneration. In future studies we will use reporter gene imaging and bioluminescence imaging to address the homing and subsequently proliferation and differentiation. On this note, molecules such as CXCR4, CXCR12 and CCL2 have been implicated in the tissue-homing ability and whether these molecules play a role needs to be further investigated.
Next, it is important to ensure the findings are replicated and relevant to the human situation. The applicability of animal data may not adequately mimic human pathophysiology as animals are often young, without any comorbidities, and are not exposed to the range of interacting interventions that humans often receive. Therefore, it is important to validate these findings in a large animal model (such as ovine) and subsequent clinical trials to optimize the timing, route, and formulation. Further studies are required to investigate the efficacy of different types of cells in cartilage defects with different diameters and depths (chondral, subchondral, osteochondral).

In summary, our findings indicate that mixed co-culture of BMSCs+ACCs can differentiate into functional cartilage-like tissues in an in vivo OA model and that these findings could potentially be developed into treatments for osteoarthritis and cartilage damage using a patient's own cells.

\section{ACKNOWLEDGMENTS}

We thank staff at the Medical Engineering Research Facility for assistance with animal care. This study was supported by Prince Charles Hospital Foundation (MS2012-25) and National Health and Medical Research Council of Australia (APP1032738).

\section{DISCLOSURE/CONFLICT OF INTEREST}

The authors declare no conflict of interest.

1. Mobasheri A, Kalamegam G, Musumeci G, et al. Chondrocyte and mesenchymal stem cell-based therapies for cartilage repair in osteoarthritis and related orthopaedic conditions. Maturitas 2014;78: 188-198.

2. Memon A, Quinlan J. Surgical treatment of articular cartilage defects in the knee: are we winning? Adv Orthop 2012;2012:528423.

3. Kock L, Donkelaar CC, Ito K. Tissue engineering of functional articular cartilage: the current status. Cell Tissue Res 2011;347:613-627.

4. Hunziker EB. The elusive path to cartilage regeneration. Adv Mater 2009;21:3419-3424.

5. Pelttari K, Winter A, Steck E, et al. Premature induction of hypertrophy during in vitro chondrogenesis of human mesenchymal stem cells correlates with calcification and vascular invasion after ectopic transplantation in SCID mice. Arthritis Rheum 2006;54:3254-3266.

6. Scotti C, Tonnarelli B, Papadimitropoulos A, et al. Recapitulation of endochondral bone formation using human adult mesenchymal stem cells as a paradigm for developmental engineering. Proc Natl Acad Sci 2010;107:7251-7256.

7. Kim Y-J, Kim H-J, Im G-I. PTHrP promotes chondrogenesis and suppresses hypertrophy from both bone marrow-derived and adipose tissue-derived MSCs. Biochem Biophys Res Commun 2008;373: 104-108.

8. Weiss $S$, Hennig T, Bock R, et al. Impact of growth factors and PTHrP on early and late chondrogenic differentiation of human mesenchymal stem cells. J Cell Physiol 2010;223:84-93.

9. Zhang $M$, Xie R, Hou W, et al. PTHrP prevents chondrocyte premature hypertrophy by inducing cyclin-D1-dependent Runx2 and Runx3 phosphorylation, ubiquitylation and proteasomal degradation. J Cell Sci 2009;122:1382-1389.

10. Guo J, Chung U-I, Yang D, et al. PTH/PTHrP receptor delays chondrocyte hypertrophy via both Runx2-dependent and-independent pathways. Dev Biol 2006;292:116-128.

11. Hong E, Reddi AH. Dedifferentiation and redifferentiation of articular chondrocytes from surface and middle zones: changes in microRNAs221/-222,-140, and-143/145 expression. Tissue Eng Part A 2013;19: 1015-1022.

12. Darling EM, Athanasiou KA. Rapid phenotypic changes in passaged articular chondrocyte subpopulations. J Orthop Res 2005;23:425-432. 
13. Fischer J, Dickhut A, Rickert M, et al. Human articular chondrocytes secrete parathyroid hormone-related protein and inhibit hypertrophy of mesenchymal stem cells in coculture during chondrogenesis. Arthritis Rheum 2010;62:2696-2706.

14. Meretoja VV, Dahlin RL, Kasper FK, et al. Enhanced chondrogenesis in co-cultures with articular chondrocytes and mesenchymal stem cells. Biomaterials 2012;33:6362-6369.

15. Qing C, Wei-ding C, Wei-min F. Co-culture of chondrocytes and bone marrow mesenchymal stem cells in vitro enhances the expression of cartilaginous extracellular matrix components. Braz J Med Biol Res 2011;44:303-310.

16. Lettry V, Hosoya K, Takagi S, et al. Coculture of equine mesenchymal stem cells and mature equine articular chondrocytes results in improved chondrogenic differentiation of the stem cells. Jpn J Vet Res 2010;58:5-15.

17. Zuo Q, Cui W, Liu F, et al. Co-cultivated mesenchymal stem cells support chondrocytic differentiation of articular chondrocytes. Int Orthop 2013;37:747-752.

18. Wu L, Leijten JC, Georgi N, et al. Trophic effects of mesenchymal stem cells increase chondrocyte proliferation and matrix formation. Tissue Eng Part A 2011;17:1425-1436.

19. Hildner F, Concaro S, Peterbauer A, et al. Human adipose-derived stem cells contribute to chondrogenesis in coculture with human articular chondrocytes. Tissue Eng Part A 2009;15:3961-3969.

20. Giovannini S, Diaz-Romero J, Aigner T, et al. Micromass co-culture of human articular chondrocytes and human bone marrow mesenchymal stem cells to investigate stable neocartilage tissue formation in vitro. Eur Cell Mater 2010;20:59.

21. Tsuchiya K, Chen G, Ushida T, et al. The effect of coculture of chondrocytes with mesenchymal stem cells on their cartilaginous phenotype in vitro. Mater Sci Eng C 2004;24:391-396.

22. Mareddy S, Crawford R, Brooke G, et al. Clonal isolation and characterization of bone marrow stromal cells from patients with osteoarthritis. Tissue Eng 2007;13:819-829.

23. Xiao Y, Mareddy S, Crawford R. Clonal characterization of bone marrow derived stem cells and their application for bone regeneration. Int J Oral Sci 2010;2:127-135.

24. Prasadam I, Friis T, Shi W, et al. Osteoarthritic cartilage chondrocytes alter subchondral bone osteoblast differentiation via MAPK signalling pathway involving ERK1/2. Bone 2010;46:226-235.

25. Prasadam I, van Gennip S, Friis $\mathrm{T}$, et al. ERK-1/2 and $\mathrm{p} 38$ in the regulation of hypertrophic changes of normal articular cartilage chondrocytes induced by osteoarthritic subchondral osteoblasts. Arthritis Rheum 2010;62:1349-1360.

26. Prasadam I, Batra J, Perry $\mathrm{S}$, et al. Systematic identification, characterization and target gene analysis of microRNAs involved in osteoarthritis subchondral bone pathogenesis. Calcified Tissue Int 2016;99:43-55.

27. Vandesompele J, De Preter K, Pattyn F, et al. Accurate normalization of real-time quantitative $\mathrm{RT}$-PCR data by geometric averaging of multiple internal control genes. Genome Biol 2002;3:RESEARCH0034.

28. Bookout $A L$, Mangelsdorf DJ. Quantitative real-time PCR protocol for analysis of nuclear receptor signaling pathways. Nucl Recept Signal 2003;1:e012.

29. Rosenberg L. Chemical basis for the histological use of safranin $O$ in the study of articular cartilage. J Bone Joint Surg Am 1971;53:69-82.

30. Rutgers $M$, van Pelt MJ, Dhert WJ, et al. Evaluation of histological scoring systems for tissue-engineered, repaired and osteoarthritic cartilage. Osteoarthritis Cartilage 2010;18:12-23.

31. Mankin HJ, Dorfman H, Lippiello $L$, et al. Biochemical and metabolic abnormalities in articular cartilage from osteo-arthritic human hips. II. Correlation of morphology with biochemical and metabolic data. J Bone Joint Surg Am 1971;53:523-537.
32. Maes C. Signaling pathways effecting crosstalk between cartilage and adjacent tissues: seminars in cell and developmental biology: the biology and pathology of cartilage. Semin Cell Dev Biol 2016;62:16-33.

33. Brittberg $M$, Lindahl $A$, Nilsson $A$, et al. Treatment of deep cartilage defects in the knee with autologous chondrocyte transplantation. N Engl J Med 1994;331:889-895.

34. Chen S, Fu P, Cong R, et al. Strategies to minimize hypertrophy in cartilage engineering and regeneration. Genes Dis 2015;2:76-95.

35. Benya PD, Shaffer JD. Dedifferentiated chondrocytes reexpress the differentiated collagen phenotype when cultured in agarose gels. Cell 1982;30:215-224.

36. Schnabel M, Marlovits S, Eckhoff G, et al. Dedifferentiation-associated changes in morphology and gene expression in primary human articular chondrocytes in cell culture. Osteoarthritis Cartilage 2002;10: 62-70.

37. Caron MM, Emans PJ, Coolsen MM, et al. Redifferentiation of dedifferentiated human articular chondrocytes: comparison of 2D and 3D cultures. Osteoarthritis Cartilage 2012;20:1170-1178.

38. Mueller MB, Tuan RS. Functional characterization of hypertrophy in chondrogenesis of human mesenchymal stem cells. Arthritis Rheum 2008;58:1377-1388.

39. Hennig T, Lorenz $H$, Thiel A, et al. Reduced chondrogenic potential of adipose tissue derived stromal cells correlates with an altered TGF $\beta$ receptor and BMP profile and is overcome by BMP-6. J Cell Physiol 2007;211:682-691.

40. Bian L, Zhai DY, Mauck RL, et al. Coculture of human mesenchymal stem cells and articular chondrocytes reduces hypertrophy and enhances functional properties of engineered cartilage. Tissue Eng Part A 2011;17:1137-1145.

41. Meretoja VV, Dahlin $\mathrm{RL}$, Wright $\mathrm{S}$, et al. Articular chondrocyte redifferentiation in $3 \mathrm{D}$ co-cultures with mesenchymal stem cells. Tissue Eng Part C Methods 2014;20:514-523.

42. Sabatino MA, Santoro R, Gueven $S$, et al. Cartilage graft engineering by co-culturing primary human articular chondrocytes with human bone marrow stromal cells. J Tissue Eng Regener Med 2015;9: 1394-1403.

43. Mapp PI, Walsh DA. Mechanisms and targets of angiogenesis and nerve growth in osteoarthritis. Nat Rev Rheumatol 2012;8:390-398.

44. Hamilton JL, Nagao M, Levine BR, et al. Targeting VEGF and its receptors for the treatment of osteoarthritis and associated pain. J Bone Miner Res 2016;31:911-924.

45. Yuan Q, Sun L, Li J-J et al. Elevated VEGF levels contribute to the pathogenesis of osteoarthritis. BMC Musculoskelet Disord 2014;15:1.

46. Tiku ML, Sabaawy HE. Cartilage regeneration for treatment of osteoarthritis: a paradigm for nonsurgical intervention. Ther Adv Musculoskelet Dis 2015;7:76-87.

47. Zhang $\mathrm{X}$, Crawford $\mathrm{R}$, Xiao Y. Chondromodulin-1 ameliorates osteoarthritis progression in vitro and in vivo. Osteoarthritis Cartilage 2015;23:A294

48. Zhang $\mathrm{L}, \mathrm{Hu} \mathrm{J}$, Athanasiou $\mathrm{KA}$. The role of tissue engineering in articular cartilage repair and regeneration. Crit Rev Biomed Eng 2009;37:1-57.

49. Bosnakovski D, Mizuno M, Kim G, et al. Chondrogenic differentiation of bovine bone marrow mesenchymal stem cells (MSCs) in different hydrogels: influence of collagen type II extracellular matrix on MSC chondrogenesis. Biotechnol Bioeng 2006;93:1152-1163.

50. Aung A, Gupta G, Majid G, et al. Osteoarthritic chondrocyte-secreted morphogens induce chondrogenic differentiation of human mesenchymal stem cells. Arthritis Rheum 2011;63:148-158.

51. Leyh M, Seitz A, Dürselen L, et al. Osteoarthritic cartilage explants affect extracellular matrix production and composition in cocultured bone marrow-derived mesenchymal stem cells and articular chondrocytes. Stem Cell Res Ther 2014;5:1. 